\title{
Research of Mechanical NVH Study
}

\author{
Qiupeng Wang ${ }^{1}$ \\ ${ }^{1}$ Xi 'an Railway Vocational and Technical College, Xi 'an, Shaanxi, 710014
}

KEYWORDS: NVH; Mechanical Security; Develop Trend

\begin{abstract}
NVH characteristic is to study how to address and reduce the mechanical operation of the noise, vibration and comfort issues. In recent years carried out against mechanical vibration and noise control technology for the latest development and trend Survey, the development of NVH technology, research methods and future trends to make paper for domestic mechanical engineering related work and provide the necessary information guide.
\end{abstract}

\section{Introduction}

Study on NVH characteristics not only applies to the entire machinery of new product development process, but also for research to improve the comfort of the existing models. This is a modeling analysis of a system or a mechanical assembly, to find the greatest impact on the comfort factor, by improving the condition of the vibration excitation source (or decrease frequency shift) or control noise and vibration excitation source to the vehicle interior transfer to improve ride comfort. Vibration Isolation of mechanical power assembly mounting system and engine intake and exhaust noise study is to improve vehicle comfort important content development and improvement of hydraulic mounting system so that this problem be a better solution. The suspension system and steering system of road irregularity excitation transfer and the response has a great influence on the driver and passenger comfort, dynamics analysis of the suspension system can improve its transmission characteristics, to reduce vibration and noise; by of the steering mechanism and finite element analysis instrument panel, steering column tube can make the natural frequency of the steering wheel out of the excitation frequency range and ensures minimum amplitude in response to the dashboard. Noise generated by the mechanical brake when a serious impact on the vehicle interior occupant comfort, proved brake noise is mainly due to the brake friction element uneven wear caused by the brake disk and other components for finite element analysis and its wear characteristics Study on the impact of noise and other issues can be improved NVH performance braking condition of the vehicle. In addition, with the continuous improvement of the speed of high-speed air flow and body shock vibration noise generated by friction it has become an important source of noise in the passenger compartment. Machinery in use for some time, resulting $\mathrm{NVH}$ characteristics of some of the elements (such as gear drive train, couplings, rubber suspension bushings, brake in the brake disk, etc.) of the vehicle will wear a major impact, they strength, reliability and sensitivity analysis is to study the characteristics of the vehicle's important work, which is the study of the mechanical properties of a so-called NVH of high mileage.

\section{Noise Mechanism Rear Axle}

Dynamic excitation gear is the root cause of vibration and noise gears, gear dynamic excitation generally consists of two parts, energized by the dynamic meshing gears itself produced called internal motivation, excited by an external system such as an engine gear Second Order, uneven 
horizontal shaft incentives and other incentives caused by external stimuli called. According to the principle of gear, the gear tooth contact point perpendicular direction no speed difference exists slide in the tangential direction and relative speed as the speed of the gear increases, the relative sliding velocity in the process of engaging the initial point to the end point of engagement after decreases and then increases, the relative speed of the intermediate position to a zero, the friction between the teeth are in this position to change direction, so that the friction force of a pulsation cycle incentive.

For an ideal gear, internal friction is the only incentive. In fact, even if no manufacture and installation errors, there will be a gear or stiffness excitation, this is because the gear contact ratio often is not an integer, and change over time period, while the number of teeth that is engaging is a periodic change, which can cause gear when the meshing stiffness occur periodically changed. Further, since the engagement surface by the force of the position, orientation, size and other changes will cause changes in mesh stiffness, incentives for meshing stiffness caused by the change called stiffness excitation, showing the elastic force term time-varying coefficients in the system vibration model, so it is a parametric excitation. Due to the inevitable presence of processing and installation errors caused the gear tooth profile deviates from the desired position, the instantaneous transmission gear ratio change occurs, resulting in impact and collision between the teeth, so called dynamic excitation error excitation. This deviation from the tooth profile error resulting excitation belong displacement excitation. Error excitation including tooth pitch error and error, other errors can be transformed into two errors.

One pair of meshing teeth in the process, since the error and elastic deformation, the bitten biting point deviation from the theoretical line meshing engagement point, will produce meshing impact, at the same time the gnawing, gnawing a point deviated from engaging line a theoretical engagement point will produce a meshing impact, in addition to the impact of biting engagement, due to a failure of gear, such as abrasion, etc. will lead to improper assembly meshing impact, meshing impact excitation of the system is a cyclical impact force, power belongs to inspire.

\section{Noise and Vibration Control of Drive Mechanism}

Active noise control is divided into control and passive control, active control and it can be considered active control, typically for a sound field space for the spatial distribution of the sound field to generate a new source of radiation to the sound field through the opposite phase to canceling noise, and then the bridge is radiated sound field, not the proper active control, the control of economic costs and service life are not yet able, after the bridge, so most of the noise control for the rear axle study focused on passive control . Noise rear axle are both structural noise, the noise control axle is mainly from the vibration control structure, and the vibration system can be divided into excitation, transmission path response of three parts, the passive control is mainly from the three aspects starting already discussed overdrive transaxle into internal motivation and external motivation, since a separate study for the rear axle can only inspire internal rear axle, so here consider reducing internal motivation, previous research results obtained final drive gear shock is the most important incentive for excitation control measures are mainly reflected in the gear parameters and processing and assembling processes.

The main parameters of gear teeth, module, pressure angle, helix angle, etc., the impact of each parameter on the meshing impact is not the same, and some influence is the opposite, in time to do the parameter optimization and matching fully considered, even consider the process quality and cost, because of changes in the parameters may change or abrasive machining equipment, so the gear to optimize the parameters is more complex, rarely used. Error excitation is a major incentive 
internal gear, thus improving the processing and assembly of precision gears to reduce the error can effectively reduce the vibration and noise of gears, and the error is divided into excitation profile error and tooth pitch errors and other errors can be transformed for these two types of errors, so in the course of processing gear, we must ensure that there is a certain tooth and pitch accuracy, only the basis of ensuring the accuracy of some of the other measures will have a damping effect, additional gear assembly error but also on the vibration and noise of gears greatly affected, parallel axis gear, coaxial and center distance will affect the normal gear of the installation of these errors will cause engagement shock.

For gear meshing impact, there are tooth meshing contact area greatly affected, currently there are two main methods to optimize the contact area, and within a diagonal gear modification. When the law is in the diagonal Cutting processing, so that the midpoint of both sides of the tooth width of the tooth surface pressure equal angles, and in the long range the rest of the tooth pressure angle range, with the entire helix angle is changed, so either concave or convex contact region are inclined at an angle to the contact, reduce the mutation, thereby reducing the impact of teeth meshing.

Transmission path from the point of view, now is doing more to improve the structure for vibration larger, stronger regional restructuring noise radiation, increasing the local stiffness, reduce the response. Change the structure includes two categories, one of which is to increase by arranging ribs, or change the direction of the trend and ribs arranged local stiffness, reduce vibration, the other is by increasing the structural parts, such as the bridge arch plus reinforcing plates, etc., from to the same effect. Predecessors in the study of the above two methods is a certain blindness, though they are carried out for strengthening the weak structure, but does not guarantee the structure will certainly strengthen the entire structure has a vibration reducing effect, even if lowering effect, but also may change the noise emission coefficient is difficult to play a role in reducing the radiated noise, in addition to making changes in the structure, a lot of the cars on the main gear unit housing plus the dynamic vibration, which is typical of the transmission path from the isolation measures, also have a good effect.

\section{NVH Control and Improvement Measures}

Noise control measures are mainly active and passive two measures; proactive steps required to reduce the noise from the noise source, such as improved mechanical structure, improve the excitation frequency, low-speed engines to reduce noise and improve the precision and quality of assembling parts, reducing movement noise, improved suspension system components, blocking the vibration transmission, improved body structure, increasing the rigidity to improve a modal, modal reduce local quantity; passive measures require to be good sound absorption, vibration absorption, sealing, noise, vibration isolation, damping material. So far, damping, sound-absorbing materials and structures in the field of mechanical noise control to obtain a very wide range of applications, such as: damping coatings, foam, constrained layer damping structure, sound-absorbing interior surfaces, as well as recent ABA wall insulation pad, and so on. These have become the main vehicle NVH performance improvement project processing means. Sheffield Institute of Technology MKLING a study by the steel, polyurethane foam layer and a damping layer composed of isolation layer arranged under mechanical cab and the passenger compartment floor to reduce internal noise, and sound effects analysis and research. Because of the high frequency noise absorption material obvious utility, low-frequency noise is ineffective to reduce low-frequency noise and low-frequency vibration is difficult, high cost of a project, take active control to reduce low frequency noise has become a major research directions. ACTIVE NOISE CONTROL is a new noise control methods 
developed in recent years, it may be in the designated area artificially purposefully generate a secondary signal to control the primary signal to achieve the purpose of noise reduction. Study of Active Control mid-enclosed space the sound field in the 1980s, the late 1980s, the development of adaptive active noise reduction techniques to achieve selective noise reduction. Japan's Nissan in 1991 on Blue Bird car began to equip the active noise reduction system that reduces interior noise 5.6dB.1987 ASKyazey published the first using the vibration control method for reducing radiated noise papers, opened the active noise control method in which the original vibration with another vibration damping achieve their superposition, vibration isolation, vibration absorption precedent purposes. Currently, the US and Australia are in a leading position in the study of active control. On the basis of NVH theory, experimental verification and experience on the part of suppliers to strengthen quality management, make clear the technical requirements of the same basic model, high-grade type the following indicators, which really formed the design standardization, quality clarifying achieve conformity of production to guide the design, production, quality improvement,

and make customers feel value for money, improve product competitiveness. Not just to reduce $\mathrm{NVH}$ noise or vibration problem is a systemic problem.

\section{Reference:}

[1] Hussien Hussien A, Shabana Ahmed A, Tsung Wei-Jiung, Et Al.Finite Element Dynamic Analysis of The Rear Axle System. Sae Paper, 1999-01-0735.

[2] Suh I S, Orzechowski J. Drivetrain Torsional And Bending Vibration for A Rwd Vehicle Interior Noise Development. Sae Paper, 2003-01-1496.

[3] Lee S K, Go S K, Yu Dongjun, Et Al. Identification And Reduction of Gear Whine Noise of The Axle System In A Passenger Van. Sae Paper, 2005-01-2302

[4] Mark A Gehringer. Application of Experimental Transfer Path Analysis And Hybrid Frf-Based Substructuring Model To Suv Axle Noise. Sae Paper, 2005-01-1833.

[5] Sun Zhaohui, Ranek Mark, Voight Michael. Variation Reduction of Axle System Nvh. Sae Paper, 2005-01-2309. 